OPEN ACCESS

Edited by:

Ting Fan Leung,

The Chinese University of

Hong Kong, China

Reviewed by:

Aleksandar Sovtic,

The Institute for Health Protection of

Mother and Child Serbia, Serbia

Kelechi Benjamin Ugonna,

Sheffield Children's Hospital,

United Kingdom

*Correspondence:

Zhen L

zhenli@hust.edu.cn

Specialty section:

This article was submitted to

Pediatric Pulmonology,

a section of the journal

Frontiers in Pediatrics

Received: 02 July 2020 Accepted: 02 September 2020 Published: 09 October 2020

Citation:

Xia W, Guo Y, Tian Z, Luo Y, Hu D,

Shao J, Li Z and Kamel IR (2020)

Clinical Features and Temporal Changes of RT-PCR and Chest CT in

COVID-19 Pediatric Patients.

Front. Pediatr. 8:579512.

doi: 10.3389/fped.2020.579512

\section{Clinical Features and Temporal Changes of RT-PCR and Chest CT in COVID-19 Pediatric Patients}

\author{
Wei Xia ${ }^{1}$, Yu Guo ${ }^{1}$, Zhiyao Tian ${ }^{1}$, Yan $\mathrm{Luo}^{2}$, Daoyu $\mathrm{Hu}^{2}$, Jianbo Shao ${ }^{1}$, Zhen $\mathrm{Li}^{2 *}$ and \\ Ihab R. Kamel ${ }^{3}$
}

1 Department of Imaging Center, Wuhan Children's Hospital (Wuhan Maternal and Child Healthcare Hospital), Tongji Medical College, Huazhong University of Science and Technology, Wuhan, China, ${ }^{2}$ Department of Radiology, Tongji Hospital, Tongji Medical College, Huazhong University of Science and Technology, Wuhan, China, ${ }^{3}$ Russell H. Morgan Department of

Radiology and Radiological Sciences, Johns Hopkins Hospital, Johns Hopkins Med Institute, Baltimore, MD, United States

Objective: This work aims to investigate the clinical features and the temporal changes of RT-PCR and CT in COVID-19 pediatric patients.

Methods: The clinical, RT-PCR, and CT features of 114 COVID-19 pediatric in-patients were retrospectively reviewed from January 21 to March 14, 2020. All patients had chest CT on admission and were identified as positive by pharyngeal swab nucleic acid test. The clinical features were analyzed, as well as the features and the temporal changes of RT-PCR and CT.

Results: Fever $(62,54 \%)$ and cough $(61,54 \%)$ were the most common symptoms. There were 34 (30\%) cases of concurrent infections. The most common imaging features on CT were ground-glass opacities (46, 40\%) and consolidation (46, 40\%). The bilateral lower lobes were the most common pattern of involvement, with 63 cases (55\%) involving one to two lobes, and in 32 (28\%) cases CT was normal. Throughout the whole duration of COVID-19 in children, the diagnostic positive rate of RT-PCR has been far higher than that of CT (all $P<0.05$ ). For RT-PCR follow-up, reliable negative results were obtained only 7 days after the onset of symptoms. Though lung involvement on chest CT progressed rapidly in several cases, lung involvement in children with COVID-19 is mild, with a median value of 2 on CT score.

Conclusions: RT-PCR is more reliable than CT in the initial diagnosis of pediatric patients with COVID-19. On follow-up, reliable negative RT-PCR results are available 7 days after the initial symptoms. The use of CT should be considered for follow-up purposes only if necessary.

Keywords: COVID-19, polymerase chain reaction, computer tomography, child, infection

\section{INTRODUCTION}

Since December 2019, a newly discovered infectious disease named COVID-19, caused by a novel coronavirus (SARS-CoV-2), has wildly spread worldwide. Millions of COVID-19 patients have been confirmed, and thousands of children are also involved in this pandemic all over the world (1-3). 
Enrollment $(\mathrm{n}=123)$

(a) all consecutive patients admitted to the hospital during Jan $21^{\text {st }}, 2020$ to Mar $14^{\text {th }}, 2020$

(b) age $<16$ Years old, inpatients;

(c) positive RT-PCR results of COVID-19;

(d) CT in the picture archiving and communication system (PACS) performed.

(e) discharged patients only.

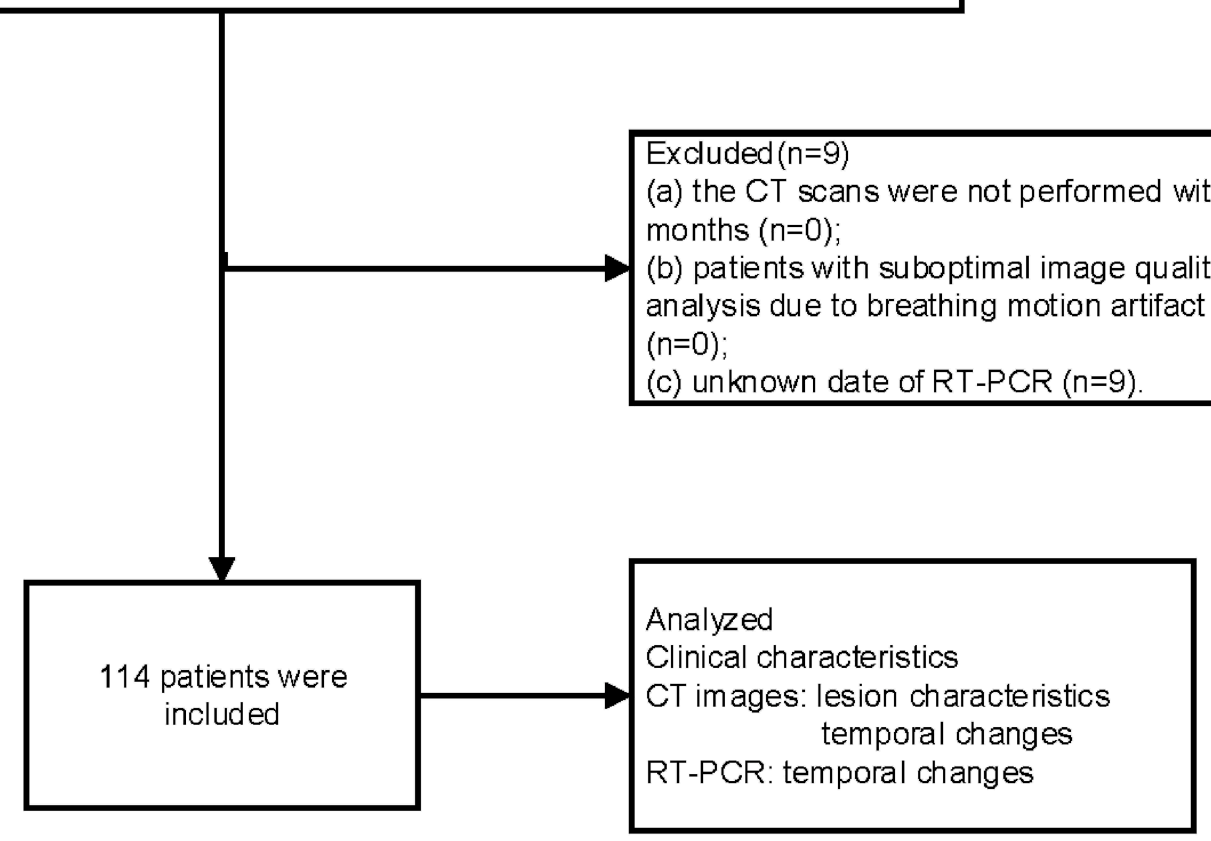

FIGURE 1 | Flow chart of this study.

To date, viral nucleic acid testing and chest CT have been considered as the main diagnostic methods for patients with suspected COVID-19 pneumonia. However, in adults, the positive rate of pharyngeal swabs reverse transcription polymerase chain reaction (RT-PCR) is only $59-61.3 \%(4,5)$. For adults, despite the possibility of false positives, the positive rate of chest CT is significantly higher than that of RT-PCR and is considered a more accurate early diagnostic tool (6-9).

For pediatric patients with suspected COVID-19, studies on chest CT values are limited $(2,10)$. Some studies have suggested that normal CT or lack of typical features were not uncommon in pediatric patients, especially during the early stage of the disease (11-14). The most common CT feature of pediatric patients was bilateral ground-glass opacities with a ratio of only $32.7 \%$ (11). However, the use of CT should be carefully assessed since pediatric patients are sensitive to radiation, and reducing CT scans for children is a top priority.

Compared with adults, the risk of severe or fatal COVID19 disease is rare, while the majority of them were mild cases requiring only conventional therapy for viral pneumonia (1113). In clinical practice, every child with fever and signs and symptoms of respiratory infection should be considered to have COVID-19, as during this pandemic healthy carriers or children with unproven COVID-19 can spread the infection to others.
In this pandemic emergency, it is important to optimize limited medical resources, reduce the radiation dose for children, and obtain a rapid and accurate diagnosis. Thus, we performed a longitudinal study to analyze the clinical characteristics, CT manifestations, and RT-PCR changes to explore early diagnosis strategies for pediatric patients.

\section{MATERIALS AND METHODS}

\section{Study Design and Participants}

The study was performed in accordance with the Declaration of Helsinki principles and good clinical practice. The study protocol was approved by the Institutional Review Board of Wuhan Children's Hospital and Tongji Hospital, Tongji Medical College, Huazhong University of Science and Technology (Wuhan, China). Written informed consent was waived because of the emergence of this infectious disease.

A medical record review was conducted using the institution's database from January 21 to March 14, 2020 in Wuhan Children's Hospital and Tongji Hospital. A prior study including only 20 patients in Wuhan Children's Hospital only described the general clinical and CT features without follow-up and did not include the evaluation of RT-PCR (12). 
TABLE 1 | Demographics of the included 114 pediatric patients.

\begin{tabular}{lc}
\hline Characteristics & Cases (percentage) \\
\hline GENDER & $69(61 \%)$ \\
Male & $45(39 \%)$ \\
Female & \\
AGE & $11(10 \%)$ \\
$<6$ months & $47(41 \%)$ \\
6 months-6 years & $56(49 \%)$ \\
$>6$ years & $42(37 \%)$ \\
DURATION FROM SYMPTOM ONSET TO DISCHARGE (DAYS) \\
8-14 & $45(39 \%)$ \\
15-21 & $15(13 \%)$ \\
$22-28$ & $12(11 \%)$ \\
$>28$ & \\
SYMPTOMS & $62(54 \%)$ \\
Fever & $61(54 \%)$ \\
Cough & $42(37 \%)$ \\
Other symptoms (sore throat, diarrhea, & $31(27 \%)$ \\
nasal discharge, sneezing, vomiting) & $3(3 \%)$ \\
CONCURRENT INFECTION & \\
None & \\
One other pathogen & \\
Two other pathogens & \\
& \\
\hline
\end{tabular}

Data are presented as cases (percentage), while percentage was calculated by the number of cases $/ 114$ cases.

The inclusion criteria were (a) age $<16$ years old, in-patients, (b) positive RT-PCR results of COVID-19, (c) CT in the picture archiving and communication system (PACS) was performed, and (d) discharged patients only.

The exclusion criteria were (a) CT scans performed earlier than 1 month, (b) patients with suboptimal image quality for analysis due to breathing motion artifact, and (c) unknown date of RT-PCR.

The pharyngeal swab samples of all the pediatric patients in our study were collected, and SARS-CoV-2 RNA was detected by RT-PCR. The RT-PCR kits were from Wuhan Huada Biotechnology Co., Ltd., Shanghai Huirui Biotechnology Co., Ltd., or Shanghai BioGerm Medical Biotechnology Co., Ltd. These were approved by China Food and Drug Administration for the detection of SARS-CoV-2 nucleic acid.

The discharge criteria were as follows: (1) normal temperature for at least 3 days, (2) significantly improved respiratory symptoms, and (3) two consecutive SARS-CoV-2 throat swabs with negative RT-PCR results, performed at least $24 \mathrm{~h}$ apart. The second result of the two consecutive RT-PCR with negative results was considered as reliable.

\section{Chest CT Protocols}

All the CT scans, including repeated CT scans, were performed according to the clinical presentation judged by pediatricians. Non-enhanced chest CT was performed in either of the four
TABLE 2 | CT characteristics of the included 114 pediatric patients.

\begin{tabular}{lc}
\hline CT characteristics & Cases (percentage) \\
\hline NUMBER OF CHEST ct PERFORMED DURING HOSPITAL STAY \\
\hline 1 & $33(29 \%)$ \\
2 & $64(56 \%)$ \\
3 & $14(12 \%)$ \\
4 & $3(3 \%)$ \\
INITIAL CT FINDINGS & \\
Normal & $32(28 \%)$ \\
Abnormal & $82(72 \%)$ \\
Unilateral lung involvement & $46(40 \%)$ \\
Bilateral lung involvement & $36(32 \%)$ \\
LOBAR INVOLVEMENT & \\
Right upper lobe & $31(27 \%)$ \\
Right middle lobe & $23(20 \%)$ \\
Right lower lobe & $43(38 \%)$ \\
Left upper lobe & $25(22 \%)$ \\
Left lower lobe & $43(38 \%)$ \\
NUMBER OF LOBES INVOLVED & \\
1 lobe & $33(29 \%)$ \\
2 lobes & $30(26 \%)$ \\
4 lobes & $8(7 \%)$ \\
5 lobes & $5(4 \%)$ \\
LESION CHARACTERISTICS & $6(5 \%)$ \\
Ground-glass opacity & \\
Consolidation & $46(40 \%)$ \\
Nodule & $46(40 \%)$ \\
Thickened interlobular septa & $7(6 \%)$ \\
\hline & $5(4 \%)$ \\
\hline
\end{tabular}

Data are presented as cases (percentage), while percentage was calculated by the number of cases/114 cases.

CT units (SOMATOM Definition AS128, Siemens; uCT 780, United Imaging; Optima 660, GE; SOMATOM Definition AS+, Siemens) with the following parameters varying according to body weight: $80-120 \mathrm{kV}, 50-120 \mathrm{mAs}$, and slice thickness of $10 \mathrm{~mm}$. The scanning range covered from the lung apex to the diaphragm on axial plane taken under free breathing, with the patients in supine position. If necessary, $0.50 \mathrm{ml} / \mathrm{kg}$ body mass of $10 \%$ chloral hydrate was taken orally before the examination. Thin-section CT images were reconstructed with $0.625-\mathrm{mm}$ collimation with a standard algorithm and then sent to the PACS for analysis.

\section{Data Collection and Analysis}

We reviewed the clinical charts of all the pediatric patients for demographic information, symptoms, date of symptom onset, admission date, discharge date, and dates and results of nucleic acid tests for COVID-19 and other identified concurrent infectious pathogens.

Two radiologists (WX and $\mathrm{ZL}$, with 12 and 18 years of experience, respectively) independently reviewed the chest CT images on PACS; only decisions reached in consensus 


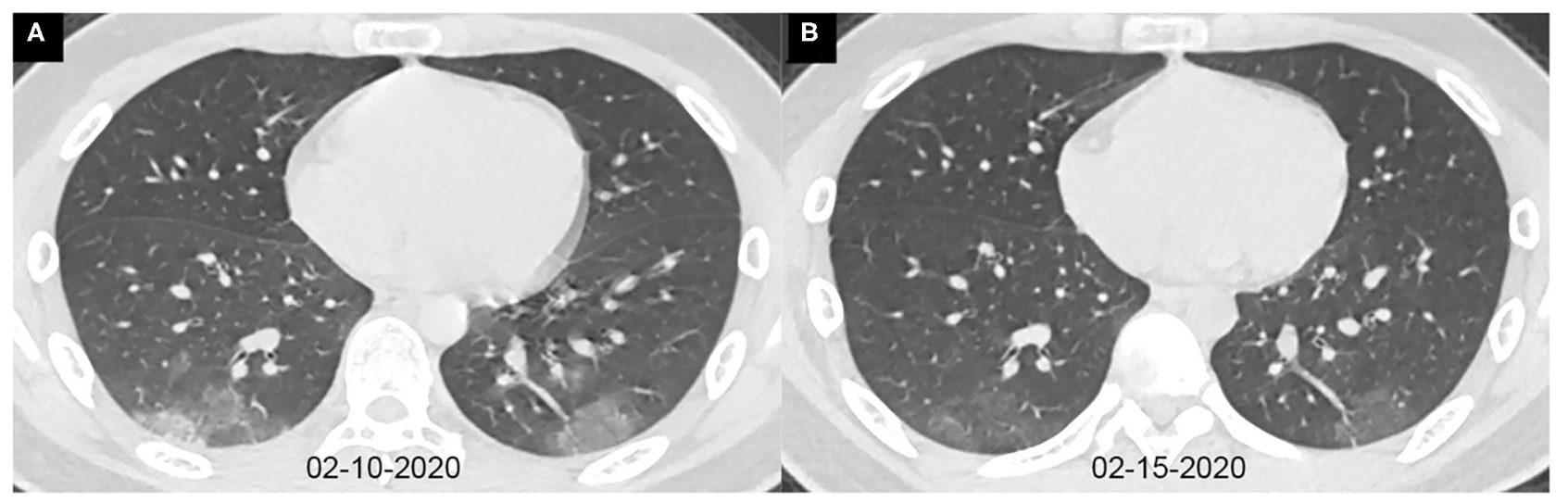

FIGURE 2 | Axial chest CT images of a 13-year-old boy with fever and cough for 10 days. Pharyngeal swab RT-PCR tests were performed on February 4, 5, and 16, 2020, with positive results, and on February 20 and 21, 2020, with negative results. Chest CTs were obtained on February 10 (A) and 15 (B). (A) The typical ground-glass opacities are shown in the bilateral lower lobes. (B) The ground-glass opacities are obviously absorbed.

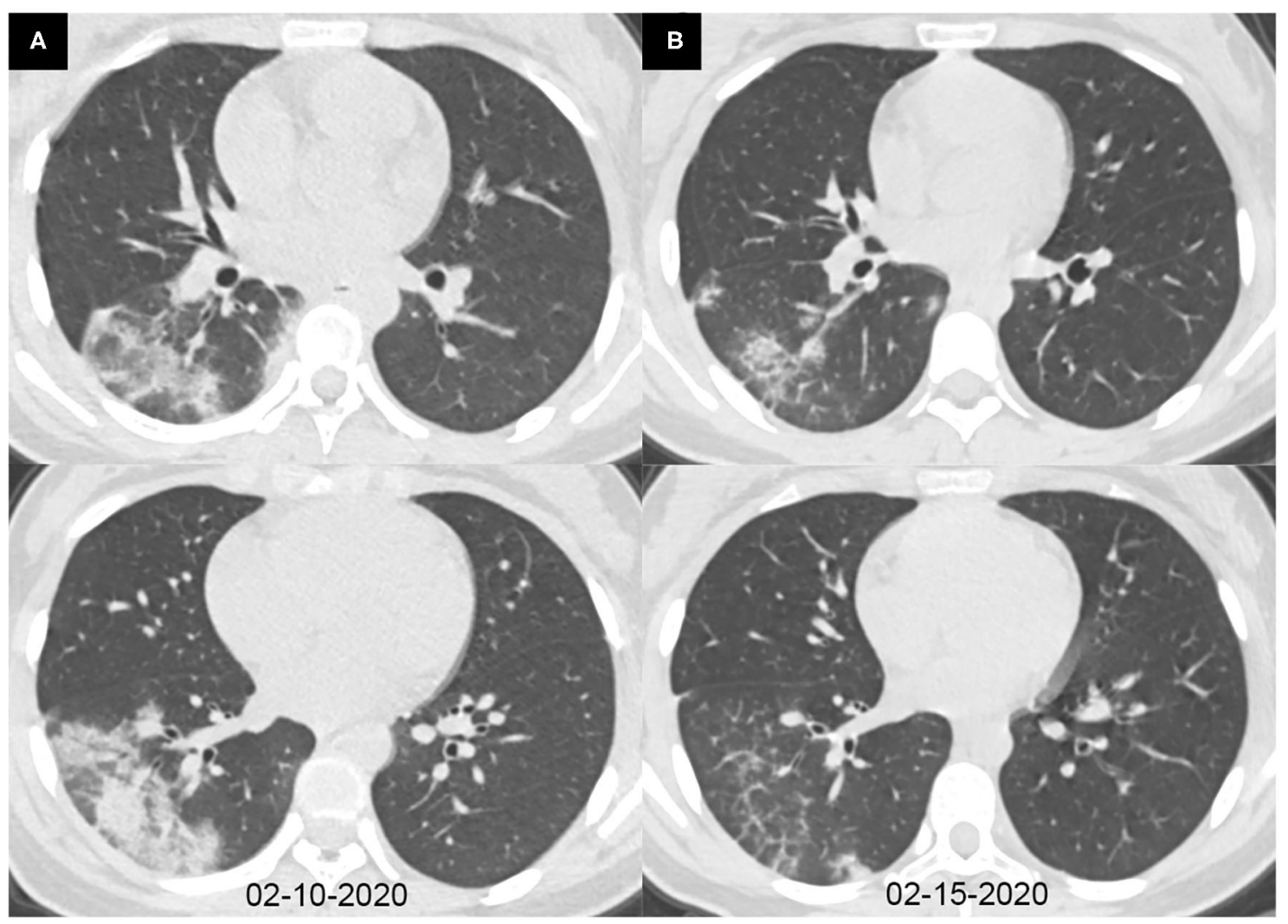

FIGURE 3 | Axial chest CT images of a 14-year-old girl with fever and cough for 6 days. Pharyngeal swab RT-PCR tests were performed on February 9, 2020, with positive results, and on February 6, 13, and 15, 2020, with negative results. Chest CTs were obtained on February 10 (A) and 15, 2020 (B). (A) Consolidations were seen in the right lower lobe. (B) The consolidations were obviously absorbed.

were reported. The initial CT images were stratified into one of two groups: normal or abnormal groups. The CT images in the abnormal group were further assessed for imaging features including (a) unilateral or bilateral distribution, (b) lobes involved, and (c) lesion characteristics. Lesion characteristics were subcategorized into (a) ground-glass 


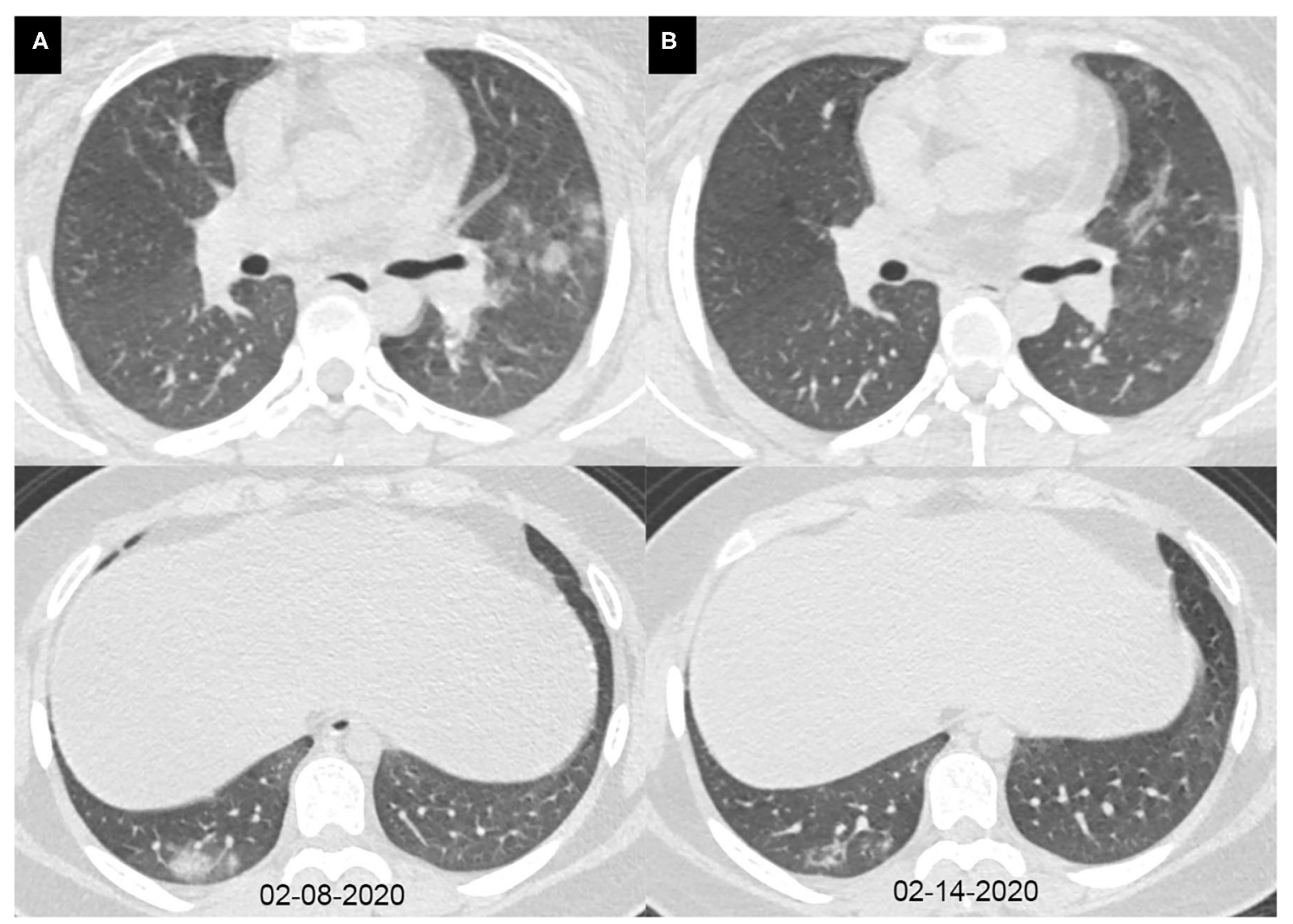

FIGURE 4 | Axial chest CT images of a 15-year-old girl with fever, cough, diarrhea, and headache for 7 days. Pharyngeal swab RT-PCR tests were performed on February 12, 2020, with positive results, and on February 15 and 17, 2020, with negative results. Chest CTs were obtained on February 8 (A) and 14, 2020 (B). (A) The subpleural consolidations and nodules were seen in the right lower lobe and the left upper lobe. (B) The consolidations and nodules were obviously absorbed.

opacity, (b) consolidation, (c) nodule, and (d) thickened interlobular septa.

The involvement of the lung was quantified according to a previously published paper, which had applied it in adults with COVID-19 (15). We divided each lung into the upper zone (above the carina), the middle zone, and the lower zone (below the inferior pulmonary vein). Each zone was scored for percentage of lung involved on a scale of $0-4$ ( 0 for $0 \%$ involvement, 1 for $<25 \%$ involvement, 2 for $25 \%$ to $<50 \%$ involvement, 3 for $50 \%$ to $<75 \%$, and 4 for more than $75 \%$ involvement). The overall CT score of lung involvement was the summation of scores from all six lung zones.

\section{Statistics}

Statistical analysis was performed using SPSS 19 (IBM Corporation, NY, USA). The day of onset of symptom was set as day 0 . The cumulative percentages of cases diagnosed by RT-PCR and CT as a function of time were plotted separately. Following an initial positive RT-PCR, the cumulative percentages on first negative results and final negative results of followup RT-PCR were plotted as well. The CT scores of lung involvement were plotted over time. The cumulative cases diagnosed by RT-PCR and CT at different time points were compared by chi-square test, two-tailed, and $P<0.05$ was considered as a statistically significant difference, as well as for the comparison of chest CT features between cases with and without co-infection.

\section{RESULTS}

Between January 21 and March 14, 2020, the total number of RT-PCR confirmed COVID-19 pediatric discharged patients who have undergone CT was 123. Of those, nine cases with an unknown date of RT-PCR were excluded. The final number of patients included in this cohort was 114. All included patients were residents of Wuhan. The flow chart is shown in Figure 1.

\section{Clinical Characteristics}

The demographics and the clinical characteristics of all the patients are summarized in Table 1. Male $(69,61 \%)$ and schoolaged children (older than 6 years; 56, 49\%) were more susceptible to COVID-19. In most cases, the duration from symptom onset to discharge was $<21$ days $(87,76 \%)$, with an average hospital 
TABLE 3 | Comparison on CT findings between 34 patients with co-infection and 80 patients without co-infection.

\begin{tabular}{|c|c|c|c|}
\hline $\begin{array}{l}\text { CT } \\
\text { characteristics }\end{array}$ & $\begin{array}{c}34 \text { cases } \\
\text { with } \\
\text { co-infection } \\
\text { (percentage) }\end{array}$ & $\begin{array}{c}80 \text { cases } \\
\text { without } \\
\text { co-infection } \\
\text { (percentage) }\end{array}$ & $P$-value \\
\hline \multicolumn{4}{|c|}{ NUMBER OF CHEST ct PERFORMED DURING HOSPITAL STAY } \\
\hline 1 & $9 / 34(26 \%)$ & 24/80 (30\%) & 0.704 \\
\hline 2 & $19 / 34(56 \%)$ & $45 / 80(56 \%)$ & 0.971 \\
\hline 3 & $6 / 34(18 \%)$ & $8 / 80(10 \%)$ & 0.409 \\
\hline 4 & $0 / 34(0 \%)$ & $3 / 80(4 \%)$ & 0.614 \\
\hline \multicolumn{4}{|c|}{ INITIAL CT FINDINGS } \\
\hline Normal & $8 / 34(24 \%)$ & $24 / 80(30 \%)$ & 0.386 \\
\hline Abnormal & $26 / 34(76 \%)$ & $56 / 80(70 \%)$ & 0.482 \\
\hline $\begin{array}{l}\text { Unilateral lung } \\
\text { involvement }\end{array}$ & $12 / 34(35 \%)$ & $34 / 80(43 \%)$ & 0.473 \\
\hline $\begin{array}{l}\text { Bilateral lung } \\
\text { involvement }\end{array}$ & 14/34(41\%) & 22/80 (27\%) & 0.151 \\
\hline \multicolumn{4}{|c|}{ LOBAR INVOLVEMENT } \\
\hline Right upper lobe & 10/34 (29\%) & $21 / 80(26 \%)$ & 0.729 \\
\hline Right middle lobe & 7/34 (21\%) & 16/80 (20\%) & 0.943 \\
\hline Right lower lobe & $15 / 34(44 \%)$ & 28/80 (35\%) & 0.358 \\
\hline Left upper lobe & 10/34 (29\%) & 15/80 (19\%) & 0.208 \\
\hline Left lower lobe & $14 / 34(41 \%)$ & 29/80 (36\%) & 0.620 \\
\hline \multicolumn{4}{|c|}{ NUMBER OF LOBES INVOLVED } \\
\hline 1 lobe & $8 / 34(24 \%)$ & 25/80 (31\%) & 0.406 \\
\hline 2 lobes & $13 / 34(38 \%)$ & $17 / 80(21 \%)$ & 0.060 \\
\hline 3 lobes & $0 / 34(0 \%)$ & $8 / 80(10 \%)$ & 0.131 \\
\hline 4 lobes & 3/34 (9\%) & 2/80 (3\%) & 0.313 \\
\hline 5 lobes & 2/34 (6\%) & $4 / 80(5 \%)$ & 1.000 \\
\hline \multicolumn{4}{|c|}{ LESION CHARACTERISTICS } \\
\hline $\begin{array}{l}\text { Ground-glass } \\
\text { opacity }\end{array}$ & $14 / 34(41 \%)$ & $32 / 80(40 \%)$ & 0.907 \\
\hline Consolidation & 15/34 (44\%) & $31 / 80$ (39\%) & 0.593 \\
\hline Nodule & 2/34 (6\%) & $5 / 80(6 \%)$ & 1.000 \\
\hline $\begin{array}{l}\text { Thickened } \\
\text { interlobular septa }\end{array}$ & $2 / 34(6 \%)$ & $3 / 80(4 \%)$ & 0.993 \\
\hline
\end{tabular}

Data are presented as cases (percentage), while percentage was calculated by the number of cases/34 cases or number of cases/80, respectively. The $P$-values comparing the differences of $C T$ findings between patients with and without co-infection are from chi-square test. $P<0.05$ was considered as a statistically significant difference.

stay of 13 days. Fever $(62,54 \%)$ and cough $(61,54 \%)$ were the most common symptoms. A concurrent infection was found in $34(30 \%)$ cases, with mycoplasma $(29,25 \%)$ being the most common concurrent infectious pathogen.

\section{Chest CT Features}

The characteristics of chest CT are reported in Table 2. During the hospitalization period, $97(85 \%)$ pediatric patients had no more than two chest CTs, while 17 (15\%) cases had three or more CT scans, with intervals of 1 to 21 days (average, 8.9 days). Among all the patients, initially normal chest CTs were found in $32(28 \%)$ cases, among whom 24 patients had undergone repeated CT scans. No more than two lobes were involved in 63
TABLE 4 | Characteristics of RT-PCR test in 114 pediatric patients.

\begin{tabular}{lc}
\hline Characteristics & Cases (percentage) \\
\hline NUMBER OF RT-PCR TESTS PERFORMED & \\
3 & $41(36 \%)$ \\
4 & $28(24 \%)$ \\
5 & $20(17 \%)$ \\
6 & $11(10 \%)$ \\
7 & $7(6 \%)$ \\
8 & $3(3 \%)$ \\
9 & $2(2 \%)$ \\
10 & $1(1 \%)$ \\
12 & $1(1 \%)$ \\
DURATION FROM FIRST POSITIVE TO FINAL NEGATIVE RESULT (DAYS) \\
$\leq 7$ & $21(18 \%)$ \\
$8-14$ & $59(52 \%)$ \\
$15-21$ & $25(22 \%)$ \\
$22-28$ & $7(6 \%)$ \\
$>28$ & $2(2 \%)$
\end{tabular}

\section{TEMPORAL TENDENCY OF ALL THE RESULTS (FROM SYMPTOM} ONSET)

Positive to negative $91(80 \%)$

Positive to negative to positive to negative 19 (17\%)

Negative to positive to negative 4 (3\%)

Data are presented as cases (percentage), while percentage was calculated by the number of cases $/ 114$ cases.

(55\%) cases. Unilateral lung involvement was found in 46 (40\%) cases, and bilateral lung involvement was found in 36 (32\%) cases. The bilateral lower lobes were most susceptible to COVID19 , with 43 cases (38\%) affected in both the left and the right lower lobes, respectively. Ground-glass opacity $(46,40 \%)$ and consolidation $(46,40 \%)$ were the most common lesion patterns (see Figures 2, 3 for details), while nodules were found in seven (6\%) cases (see Figure 4 for details). A comparison of chest CT features between cases with and without co-infection is shown in Table 3.

\section{RT-PCR Characteristics}

Detailed information on RT-PCR is displayed in Table 4. All the cases have three to 12 times RT-PCR during their hospital stay, and $89(77 \%)$ cases have five or less RT-PCR assays, with intervals of 1-13 days (average, 3.5 days). The duration from the first positive result to the final negative result was within 21 days in 105 (92\%) cases. The most common temporal tendency pattern of RT-PCR was positive to negative (91, 80\%).

\section{Temporal Changes of Initial Chest CT and RT-PCR}

The cumulative percentage of cases identified by RT-PCR and initial chest CT is shown in Figure 5. In the first week after the onset of symptoms, the cumulative percentage of RT-PCR and CT both increased rapidly. On the 4th day after the onset of symptoms, 72 (63\%) patients had a positive RT-PCR, but only 36 $(42 \%)$ patients had infiltrates in the chest CT at the same time $\left(\chi^{2}\right.$ 


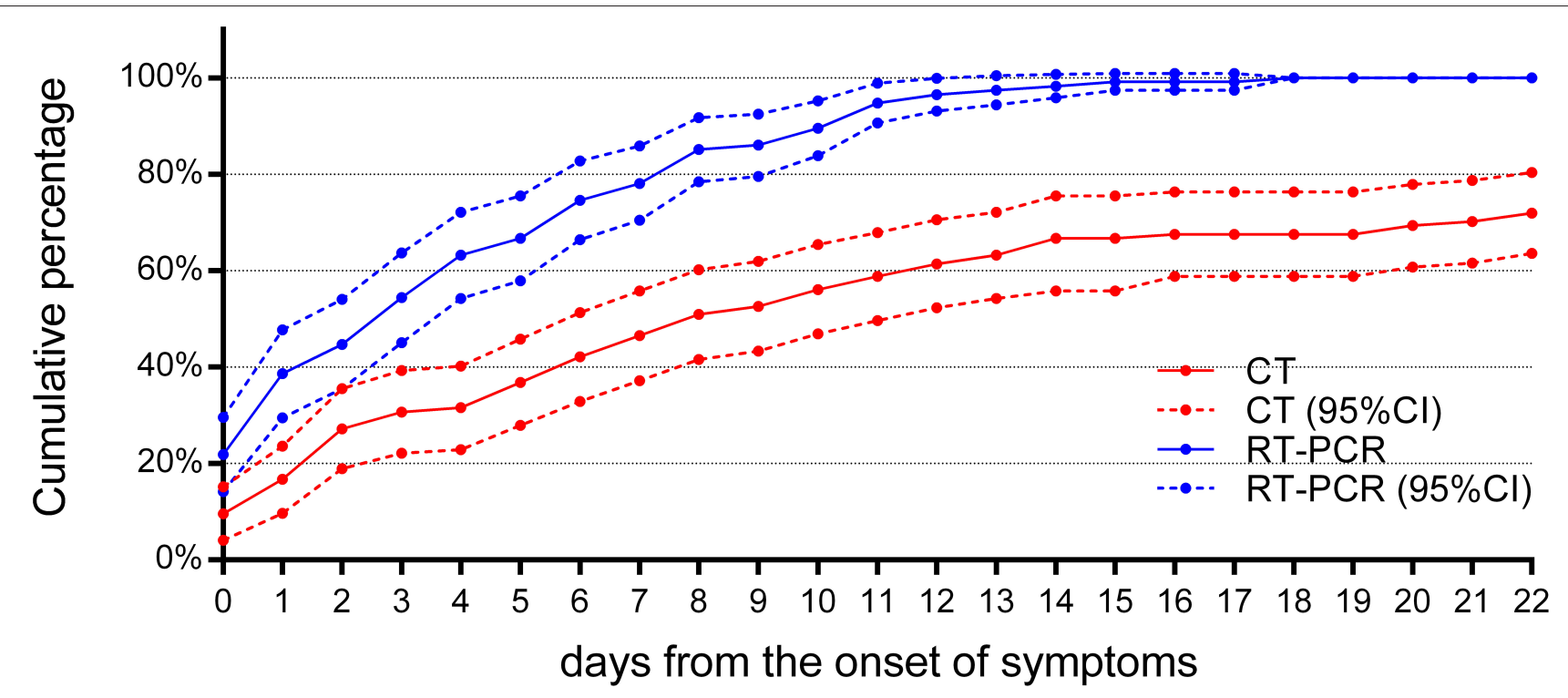

FIGURE 5 | The cumulative percentage of identified cases by RT-PCR and chest CT. Both curves of RT-PCR and CT increased rapidly after the onset of symptoms ( $n$ $=114)$. By the 7 th day after the onset of symptoms, $89(78 \%)$ cases have been confirmed by RT-PCR, compared to $53(46 \%)$ cases by chest CT $(P<0.05)$. By day 14 after the onset of symptoms, RT-PCR diagnosed 112 (98\%) cases, but chest CT was positive in only $76(67 \%)$ cases $(P<0.05)$. 95\% Cl, 95\% confidence interval.

$=22.8, P<0.05)$. On the 7 th day after the onset of symptoms, RT-PCR confirmed 89 cases (78\%) of COVID-19 pneumonia, while chest CT confirmed only 53 cases $(46 \%)\left(\chi^{2}=24.2, P<\right.$ $0.05)$. By the 14 th day of symptom onset, 112 cases $(98 \%)$ were confirmed by RT-PCR, but only 76 cases (67\%) were positive for chest CT $\left(\chi^{2}=39.3, P<0.05\right)$. At 18 days after the onset of symptoms, the last patient was confirmed by RT-PCR, compared to $77(68 \%)$ identified cases by CT $\left(\chi^{2}=44 \cdot 2, P<0.05\right)$. As the chest CT of 32 children was normal, the cumulative percentage of cases identified by CT was only $72 \%$ (82), compared to 114 (100\%) cases identified by RT-PCR $\left(\chi^{2}=37.2, P<0.05\right)$, by day 22 after the onset of symptoms. Throughout the whole duration of COVID-19 in children, the diagnostic positive rate of RT-PCR has been far higher than that of CT (all $P<0.05$ ), as shown in Table 5.

At the same time, it was found that, after the first week of symptoms, the rise of cumulative percentage of cases identified by RT-PCR and chest CT became less and reached a plateau after 11 days. Compared with the initial chest CT, the cumulative percentage of cases identified by RT-PCR is more significant.

\section{Temporal Changes of RT-PCR and Chest CT}

For treatment response, a review of the follow-up RT-PCR for children indicated that (Figure 6) there were no reliable negative RT-PCR results until 7 days after the onset of symptoms; otherwise, it should be false negative. There were $52(46 \%)$ reliable negative RT-PCR result cases on the 14th day, 92 (81\%) cases on the 21st day, and 106 (93\%) cases on the 28th day, and the latest time for RT-PCR to turn negative was 37 days after the onset of symptoms.
A total of 215 chest CTs were obtained from 114 children with COVID-19. In Figure 7, the CT scores on lung involvement were plotted as a function of time. The CT score reached 14 in one case during $0-5$ days from the onset of symptoms, but the median values of the CT scores were low before the 11th day from the onset of symptoms, with a median value of 1 . The median value of CT score on lung involvement reached a peak at 12-17 days, with a median value of 2 . After the 24 th day from the onset of symptoms, the median value of CT score fell back to 1 .

\section{DISCUSSION}

In the current study, the length of hospital stay in most pediatric patients with COVID-19 (average, 13 days) was not significantly different from pneumonia caused by other pathogens (average, 11.2 days) (16). Interestingly, the number of pediatric patients younger than 6 months was significantly less than that in other age groups, which may be related to residual protection from maternal immune factors, such as virus-specific antibodies according to Zeng et al. (17). The number of patients in two other age groups was not significantly different from each other. Similar to results reported in previous studies, according to lung involvement, the majority of cases were mild cases $(11,12,18)$. Severe and fatal cases in pediatric patients were very rare. Thus, except for treatment for individuals, early diagnosis to avoid the further spread of the disease was an essential issue under this pandemic circumference.

In the current study, chest CT had similar characteristic manifestations including ground-glass opacities and consolidations with bilateral lower lobes, as was recently published $(12,19)$. Of all COVID-19-positive children in this study, completely normal chest CT was not uncommon, 
infiltrates on chest CT were not severe in most of them, and concurrent infections may lead to ambiguous CT imaging. Similar to previous studies, CT of the chest is often atypical, especially in the early stage, resulting in difficulty in diagnosing or ruling out COVID-19 (20). In addition, in our study, the cumulative percentage of identified cases by chest CT was low throughout the course of COVID-19, compared to RT-PCR. This is totally different from the results of related research in adults. In adults, as an important complementary tool for less-sensitive and time-consuming RT-PCR, chest CT has first been considered as a diagnostic tool for clinically confirmed cases of COVID-19 in China $(21,22)$. Considering that the clinical manifestations and CT features of most children are mild, CT has limited diagnostic value for children (especially 0-7 days after onset). Therefore, CT of the chest is of limited value in diagnostic algorithm and should be discouraged to reduce the radiation dose to children.

In the current study, throughout the entire course of COVID19 pneumonia in children, the diagnostic positive rate of CT has been far lower than that of RT-PCR (all $P<0.05$ ), and 28\% of children have no obvious abnormal signs of CT. However, in adults, previous studies have shown that the positive rate of RT-PCR is only $59-61.3 \%$, while the positive rate of chest CT is $88 \%(4,5)$. These findings suggest that most infected pediatric patients have less lung involvement in the early stages of COVID19 infection. As some COVID-19 cases confirmed by RT-PCR could have no lesions on chest CT, pathogen identification by RT-PCR has a more important role in the management of an infectious source. RT-PCR may be more reliable than CT in pediatric patients' diagnosis, and repeated RT-PCR every other day is the recommended screening for pediatric patients during the first 7 days.

In addition, the main indicator of children's discharge criteria is to determine the reliable RT-PCR negative examination results. As inappropriate sampling, preservation, and processing may lead to a low virus level, we may inevitably get false negative RT-PCR results in treatment response evaluation. Our research shows that negative RT-PCR results obtained 7 days after the onset of symptoms are reliable. This indicates that, for children whose clinical symptoms have disappeared and who may totally recover, follow-up tests of RT-PCR must be performed at least after day 7 to evaluate the efficacy.

To reduce radiation dose among the pediatric patients included in this study, most cases (85\%) had one or two chest CT scans during their hospital stay. In our study, it was found that the median values of CT scores were low before the 11th day from the onset of symptoms, with a median value of 1 . The median value of CT score on lung involvement reached a peak at 12-17 days, with a median value of 2 . After the 24 th day from onset of symptoms, the median value of CT score fell back to 1 . Compared to the median CT score of 5 on lung involvement in adults reported in a previous study (15), it indicates that children with COVID-19 pneumonia are relatively mild. As reported, lung involvement peaked on 6-11 days from symptom onset in adults, while the delayed peaking in children may be related to a different immune reaction to the virus (15). In our study, the CT score reached 14 in one case during $0-5$
TABLE 5 | Comparison of identified cases by RT-PCR and chest CT over time.

\begin{tabular}{|c|c|c|c|}
\hline $\begin{array}{c}\text { Days from } \\
\text { symptom } \\
\text { onset } \\
\text { (days) }\end{array}$ & Chest CT & RT-PCR & $P$-value \\
\hline & $\begin{array}{l}\text { Cases (percentage, } \\
95 \% \mathrm{Cl} \text { ) }\end{array}$ & $\begin{array}{l}\text { Cases (percentage, } 95 \% \\
\mathrm{Cl} \text { ) }\end{array}$ & \\
\hline 0 & $11 / 114$ (10\%, 4-15\%) & 25/114 (22\%, 14-30\%) & 0.011 \\
\hline 1 & 19/114 (17\%, 10-24\%) & 44/114 (39\%, 30-48\%) & $<0.001$ \\
\hline 2 & $31 / 114$ (27\%, 19-36\%) & $51 / 114$ (45\%, 36-54\%) & 0.006 \\
\hline 3 & $35 / 114(31 \%, 22-39 \%)$ & $62 / 114(54 \%, 45-64 \%)$ & $<0.001$ \\
\hline 4 & $36 / 114$ (32\%, 23-40\%) & 72/114 (63\%, 54-72\%) & $<0.001$ \\
\hline 5 & $42 / 114(37 \%, 28-46 \%)$ & 76/114 (67\%, 58-76\%) & $<0.001$ \\
\hline 6 & 48/114 (42\%, 33-51\%) & 85/114 (75\%, 66-83\%) & $<0.001$ \\
\hline 7 & 53/114 (46\%, 37-56\%) & 89/114 (78\%, 70-86\%) & $<0.001$ \\
\hline 8 & $58 / 114$ (51\%, 42-60\%) & 97/114 (85\%, 78-92\%) & $<0.001$ \\
\hline 9 & $60 / 114$ (53\%, 43-62\%) & 98/114 (86\%, 80-92\%) & $<0.001$ \\
\hline 10 & $64 / 114$ (56\%, 47-65\%) & 102/114 (89\%, 84-95\%) & $<0.001$ \\
\hline 11 & 67/114 (59\%, 50-68\%) & 108/114 (95\%, 91-99\%) & $<0.001$ \\
\hline 12 & 70/114 (61\%, 52-71\%) & 110/114 (96\%, 93-100\%) & $<0.001$ \\
\hline 13 & 72/114 (63\%, 54-72\%) & 111/114 (97\%, 94-100\%) & $<0.001$ \\
\hline 14 & 76/114 (67\%, 58-76\%) & $112 / 114(98 \%, 96-101 \%)$ & $<0.001$ \\
\hline 15 & 76/114 (67\%, 58-76\%) & 113/114 (99\%, 97-101\%) & $<0.001$ \\
\hline 16 & 77/114 (68\%, 59-76\%) & $113 / 114$ (99\%, 97-101\%) & $<0.001$ \\
\hline 17 & 77/114 (68\%, 59-76\%) & 113/114 (99\%, 97-101\%) & $<0.001$ \\
\hline 18 & 77/114 (68\%, 59-76\%) & $114 / 114(100 \%, 100-100 \%)$ & $<0.001$ \\
\hline 19 & 77/114 (68\%, 59-76\%) & $114 / 114(100 \%, 100-100 \%)$ & $<0.001$ \\
\hline 20 & 79/114 (69\%, 61-78\%) & $114 / 114(100 \%, 100-100 \%)$ & $<0.001$ \\
\hline 21 & 80/114 (70\%, 62-79\%) & $114 / 114(100 \%, 100-100 \%)$ & $<0.001$ \\
\hline 22 & $82 / 114(72 \%, 64-80 \%)$ & $114 / 114(100 \%, 100-100 \%)$ & $<0.001$ \\
\hline
\end{tabular}

Data are presented as cases (percentage), while percentage was calculated by the number of cases/114 cases. $P$-values comparing the differences of cumulative diagnosed cases between RT-PCR and CT at different time points are from chi-square test. $P<0.05$ was considered as a statistically significant difference. $95 \% \mathrm{Cl}$, 95\% confidence interval.

days from the onset of symptoms, which suggested that rapid progression could also be observed in pediatric patients, even if it was rare.

There are several limitations in our study. First, even if the sample was the largest as we know, the overall cases in the two included hospitals were still limited. Second, as a retrospective study, selection bias could not be avoided. Third, during this outbreak period of COVID-19, delay in seeking care (more than 7 days from symptom onset) would influence the diagnosis and the prognosis.

In conclusion, chest $\mathrm{CT}$ is not recommended as a primary method for early diagnosis in children with COVID-19, especially to avoid repeated CT scans. While RT-PCR may have a more valuable position, repeated RT-PCR every other day is the recommended screening for pediatric patients during the first 7 days. For treatment response, reliable negative RT-PCR followup results, in accordance with discharge criteria, are not available until at least 7 days after the onset of symptoms. CT can be employed as a tool to assess lung involvement only if necessary. 


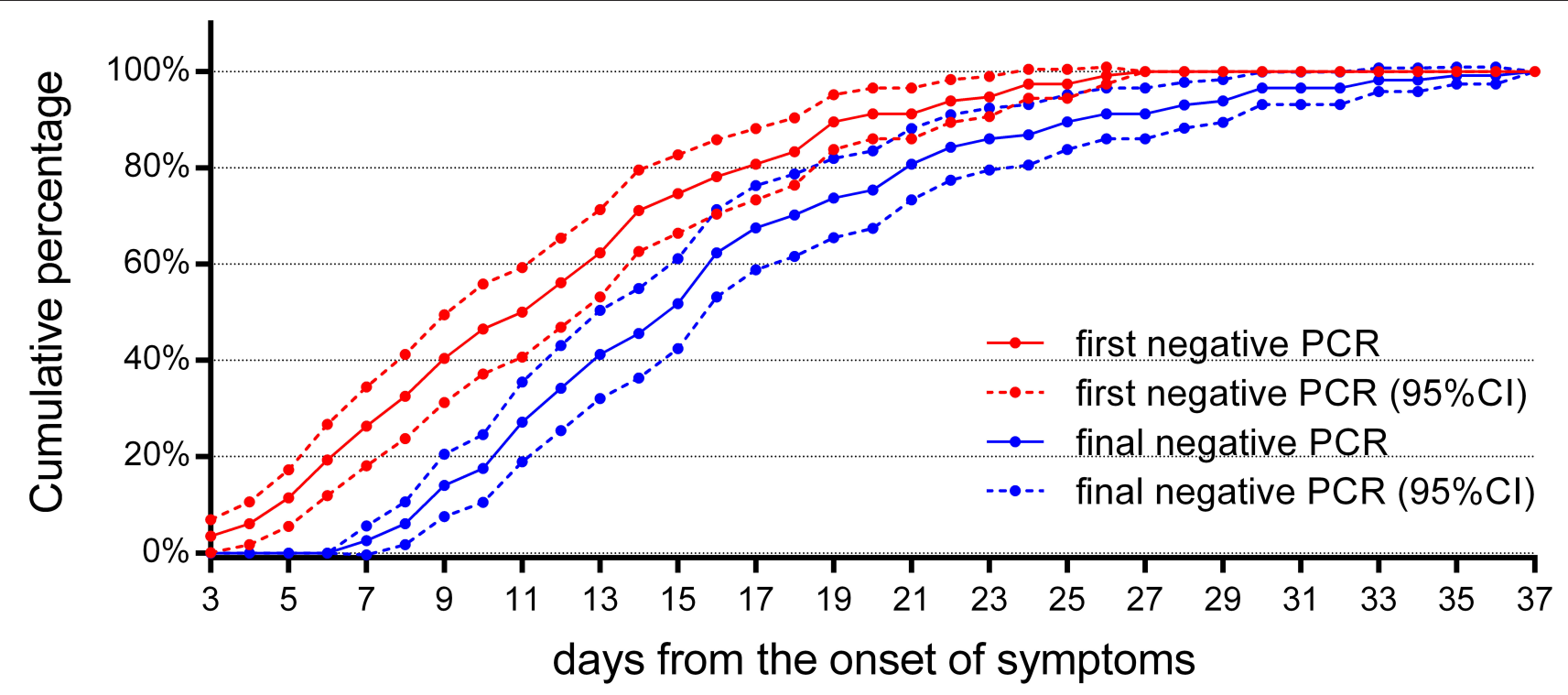

FIGURE 6 | The cumulative percentages of the follow-up RT-PCR. The cumulative percentages of the first and the final negative results of follow-up RT-PCR $(n=$ 114). The first negative RT-PCR results could be obtained as early as 3 days from the onset of symptoms in four cases, but they returned to positive later. A reliable final negative RT-PCR result could not be obtained until 7 days after the onset of symptoms. 95\% Cl, 95\% confidence interval.

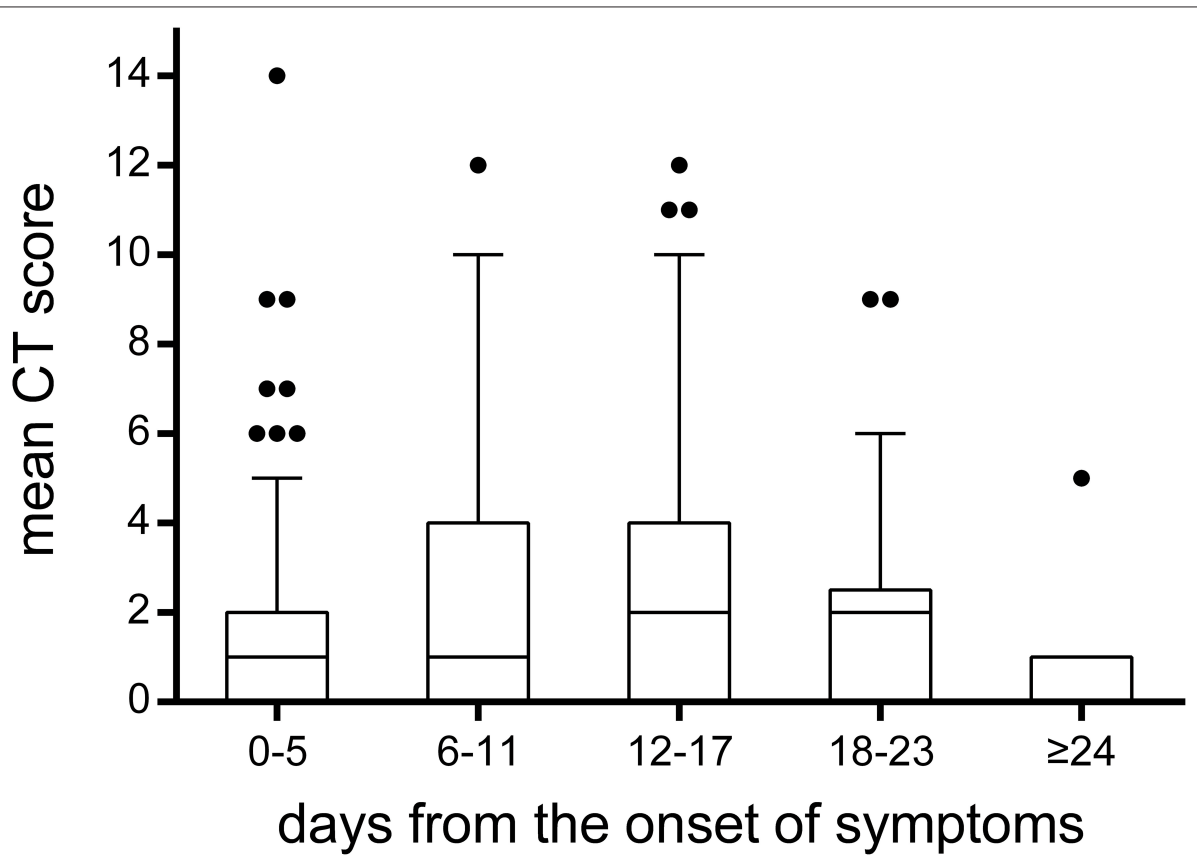

FIGURE 7 | The temporal change of CT scores on lung involvement. The CT scores on lung involvement in all chest CT scans ( $n=215)$. Throughout the whole duration of COVID-19 in children, the median value of CT scores was low, with a median value of 1 or 2 . It was worth noting that the CT score reached 14 in one case during 0-5 days from the onset of symptoms. The bounds of the boxes represent the 25 th to the 75 th percentiles; the line in the middle of the box is plotted as the median. The whiskers and the outliers are drawn according to Tukey method.

\section{DATA AVAILABILITY STATEMENT}

All datasets generated for this study are included in the article/supplementary material.

\section{ETHICS STATEMENT}

The studies involving human participants were reviewed and approved by Institutional Review Board of Wuhan Children's 
Hospital and Tongji Hospital, Tongji Medical College, Huazhong University of Science and Technology. Written informed consent from the participants' legal guardian/next of kin was not required to participate in this study in accordance with the national legislation and the institutional requirements.

\section{AUTHOR CONTRIBUTIONS}

WX, ZL, and DH conceived and designed the study. YG, YL, and $\mathrm{DH}$ contributed to the literature search. WX, YG, and YL collected the data. WX and YG analyzed the data. WX drafted the manuscript. ZL and IK revised the manuscript. WX and ZL had full access to all data in the study and took responsibility for the integrity of data and the accuracy of the

\section{REFERENCES}

1. Huang C, Wang Y, Li X, Ren L, Zhao J, Hu Y, et al. Clinical features of patients infected with 2019 novel coronavirus in Wuhan, China. Lancet. (2020) 395:497-506. doi: 10.1016/S0140-6736(20)30183-5

2. Guan W-j, Ni Z-y, Hu Y, Liang W-h, Ou C-q, He J-X, et al. Clinical characteristics of coronavirus disease 2019 in China. N Engl J Med. (2020) 382:1708-20. doi: 10.1056/NEJMoa2002032

3. Kandel N, Chungong S, Omaar A, Xing J. Health security capacities in the context of COVID-19 outbreak: an analysis of international health regulations annual report data from 182 countries. Lancet. (2020) 395:104753. doi: 10.1016/S0140-6736(20)30553-5

4. Ai T, Yang Z, Hou H, Zhan C, Chen C, Lv W, et al. Correlation of chest CT and RT-PCR testing in coronavirus disease 2019 (COVID-19) in China: a report of 1014 Cases. Radiology. (2020) 296:E32-40. doi: 10.1148/radiol.2020200642

5. Yang Y, Yang M, Shen C, Wang F, Yuan J, Li J, et al. Evaluating the accuracy of different respiratory specimens in the laboratory diagnosis and monitoring the viral shedding of 2019-nCoV infections. medRxiv [Preprint]. (2020). doi: 10.1101/2020.02.11.20021493

6. Bernheim A, Mei X, Huang M, Yang Y, Fayad ZA, Zhang N, et al. Chest CT findings in coronavirus disease-19 (COVID-19): relationship to duration of infection. Radiology. (2020) 295:200463. doi: 10.1148/radiol.2020200463

7. Fang Y, Zhang H, Xie J, Lin M, Ying L, Pang P, et al. Sensitivity of chest CT for COVID-19: comparison to RT-PCR. Radiology. (2020) 296:E115-7. doi: 10.1148/radiol.2020200432

8. Pan F, Ye T, Sun P, Gui S, Liang B, Li L, et al. Time course of lung changes on chest CT during recovery from 2019 novel coronavirus (COVID-19) pneumonia. Radiology. (2020) 295:715-21. doi: 10.1148/radiol.2020200370

9. Xie X, Zhong Z, Zhao W, Zheng C, Wang F, Liu J. Chest CT for typical 2019$\mathrm{nCoV}$ pneumonia: relationship to negative RT-PCR testing. Radiology. (2020) 296:E41-5. doi: 10.1148/radiol.2020200343

10. Wu Z, McGoogan JM. Characteristics of and important lessons from the coronavirus disease 2019 (COVID-19) outbreak in China: summary of a report of 72,314 cases from the Chinese center for disease control and prevention. JAMA. (2020) 323:1239-42. doi: 10.1001/jama.2020.2648

11. Lu X, Zhang L, Du H, Zhang J, Li YY, Qu J, et al. SARS-CoV-2 infection in children. N Engl J Med. (2020) 382:1663-5. doi: 10.1056/NEJMc2005073

12. Xia W, Shao J, Guo Y, Peng X, Li Z, Hu D. Clinical and CT features in pediatric patients with COVID-19 infection: different points from adults. Pediatr Pulmonol. (2020) 55:1169-74. doi: 10.1002/ppul.24718

13. Liu W, Zhang Q, Chen J, Xiang R, Song H, Shu S, et al. Detection of covid19 in children in early january 2020 in Wuhan, China. N Engl J Med. (2020) 382:1370-1. doi: 10.1056/NEJMc2003717 data analysis. All authors reviewed and approved the final version of the manuscript. All authors contributed to the article and approved the submitted version.

\section{FUNDING}

This research was supported by the National Natural Science Foundation of China (81771801), Hubei Province Health and Family Planning Scientific Research Project (WJ2018H0160), HUST Innovation Project 2020 (2020kfyXGYJ031), and Wuhan Children's Hospital Foundation (2020FE002). The funding organization had no role in study design, data collection, data analysis, and manuscript preparation and publication decision. This work was the responsibility of the authors.

14. Yu N, Li W, Kang Q, Xiong Z, Wang S, Lin X, et al. Clinical features and obstetric and neonatal outcomes of pregnant patients with COVID-19 in Wuhan, China: a retrospective, single-centre, descriptive study. Lancet Infect Dis. (2020) 20:559-64. doi: 10.1016/S1473-3099(20) 30176-6

15. Wang Y, Dong C, Hu Y, Li C, Ren Q, Zhang X, et al. Temporal changes of CT findings in 90 patients with COVID-19 pneumonia: a longitudinal study. Radiology. (2020) 296:E55-64. doi: 10.1148/radiol.2020200843

16. Wen ZH, Wei J, Xue HL, Chen YQ, Melnick D, Gonzalez J, et al. Epidemiology, microbiology, and treatment patterns of pediatric patients hospitalized with pneumonia at two hospitals in China: a patient chart review study. Therap Clin Risk Manag. (2018) 14:501-10. doi: 10.2147/TCRM.S143266

17. Zeng H, Xu C, Fan J, Tang Y, Deng Q, Zhang W, et al. Antibodies in infants born to mothers with COVID-19 pneumonia. JAMA. (2020) 323:1848-9. doi: 10.1001/jama.2020.4861

18. Zhu N, Zhang D, Wang W, Li X, Yang B, Song J, et al. A novel coronavirus from patients with pneumonia in China, 2019. N Engl J Med. (2020) 382:72733. doi: 10.1056/NEJMoa2001017

19. Chung M, Bernheim A, Mei X, Zhang N, Huang M, Zeng X, et al. CT imaging features of 2019 novel coronavirus (2019-nCoV). Radiology. (2020) 295:202-7. doi: 10.1148/radiol.2020200230

20. Virkki R, Juven T, Rikalainen H, Svedstrom E, Mertsola J, Ruuskanen O. Differentiation of bacterial and viral pneumonia in children. Thorax. (2002) 57:438-41. doi: 10.1136/thorax.57.5.438

21. General Office of the National Health Commission of China. Diagnosis and Treatment Protocol for 2019-nCoV (Fifth Trial Edition). (2020). Available online at: http://www.nhc.gov.cn/yzygj/s7653p/202002/ d4b895337e19445f8d728fcaf1e3e13a.shtml (accessed June 24, 2020).

22. Yang W, Yan F. Patients with RT-PCR confirmed COVID-19 and normal chest CT. Radiology. (2020) 295:E3. doi: 10.1148/radiol.2020200702

Conflict of Interest: The authors declare that the research was conducted in the absence of any commercial or financial relationships that could be construed as a potential conflict of interest.

Copyright (c) 2020 Xia, Guo, Tian, Luo, Hu, Shao, Li and Kamel. This is an openaccess article distributed under the terms of the Creative Commons Attribution License (CC BY). The use, distribution or reproduction in other forums is permitted, provided the original author(s) and the copyright owner(s) are credited and that the original publication in this journal is cited, in accordance with accepted academic practice. No use, distribution or reproduction is permitted which does not comply with these terms. 